\title{
UK Renal Registry 11th Annual Report (December 2008): Appendix G Coding: Ethnicity; EDTA primary renal diagnoses and EDTA causes of death
}

\section{G1: Ethnicity coding}

Ethnicity data is recorded in the clinical information systems in the individual renal centres in the format of 9S... read codes.

\begin{tabular}{|c|c|c|c|c|}
\hline Ethnic category & Read code & Old PAS & Renal Assoc & New PAS \\
\hline White & 9S1.. & 0 & $\mathrm{~W}$ & A1 \\
\hline Black Caribbean & $9 \mathrm{~S} 2 .$. & 1 & & M1 \\
\hline Black African & 9S3.. & 2 & & N1 \\
\hline Black other/non-mixed origin & 9S4.. & 3 & & P1 \\
\hline Indian & 9S6.. & 4 & & H1 \\
\hline Pakistani & 9S7.. & 5 & & $\mathrm{~J} 1$ \\
\hline Bangladeshi & 9S8.. & 6 & & K1 \\
\hline Chinese & $9 S 9 .$. & 7 & $\mathrm{C}$ & R1 \\
\hline Black British & $9 S 41$. & & & $\mathrm{PD}$ \\
\hline Black Caribbean & $9 S 42$. & & & \\
\hline Black North African & $9 \mathrm{~S} 43$. & & & \\
\hline Black other African country & $9 S 44$. & & & \\
\hline Black East African Asian & $9 S 45$. & & & \\
\hline Black Indian sub-continent & $9 S 46$. & & & \\
\hline Black other Asian & $9 S 47$. & & & \\
\hline Black Black other & $9 S 48$. & & $\mathrm{B}$ & $\mathrm{PE}$ \\
\hline Black other/mixed & 9S5.. & & & \\
\hline Other Black Black/White origin & 9S51. & & & GC \\
\hline Other Black Black/Asian origin & 9S52. & & & GA \\
\hline Other ethnic non-mixed (NMO) & 9SA.. & & & \\
\hline Brit. ethnic minor. spec. (NMO) & 9SA1. & & & \\
\hline Brit. ethnic minor. unsp (NMO) & 9SA2. & & & \\
\hline Caribbean Island (NMO) & 9SA3. & & & \\
\hline North African Arab (NMO) & 9SA4. & & & \\
\hline Other African countries (NMO) & 9SA5. & & & \\
\hline East African Asian (NMO) & 9SA6. & & & \\
\hline Indian sub-continent (NMO) & 9SA7. & & & \\
\hline Other Asian (NMO) & 9SA8. & & A & L1 \\
\hline Irish (NMO) & 9SA9. & & & B1 \\
\hline Greek Cypriot (NMO) & 9SAA. & & & CG \\
\hline Turkish Cypriot (NMO) & 9SAB. & & & CJ \\
\hline Other European (NMO) & 9SAC. & & & $\mathrm{C} 1$ \\
\hline
\end{tabular}

\section{KARGER}

Fax +4161306 1234

E-Mail karger@karger.ch

www.karger.com
UK Renal Registry, Southmead Hospital, Southmead Road, Bristol BS10 5NB, UK

Email: renalreg@renalreg.com 


\begin{tabular}{|c|c|c|c|c|}
\hline Ethnic category & Read code & Old PAS & Renal Assoc & New PAS \\
\hline Other ethnic mixed origin & 9SB.. & 8 & & \\
\hline Other ethnic Asian/White origin & 9SB2. & & & F1 \\
\hline Other ethnic mixed white origin & 9SB3. & & & \\
\hline Other ethnic other mixed origin & 9SB4. & & & G1 \\
\hline
\end{tabular}

\section{G2: EDTA primary renal diagnoses}

\begin{tabular}{|c|c|c|}
\hline Code & Title & Group \\
\hline 0 & Chronic renal failure; aetiology uncertain unknown/unavailable & Uncertain \\
\hline 10 & Glomerulonephritis; histologically NOT examined & Uncertain \\
\hline 11 & Focal segmental glomeruloscerosis with nephrotic syndrome in children & Glomerulonephritis \\
\hline 12 & IgA nephropathy (proven by immunofluorescence, not code 76 and not 85) & Glomerulonephritis \\
\hline 13 & $\begin{array}{l}\text { Dense deposit disease; membrano-proliferative GN; type II (proven by immunofluorescence } \\
\text { and/or electron microscopy) }\end{array}$ & Glomerulonephritis \\
\hline 14 & Membranous nephropathy & Glomerulonephritis \\
\hline 15 & $\begin{array}{l}\text { Membrano-proliferative GN; type I (proven by immunofluorescence and/or electron } \\
\text { microscopy - not code } 84 \text { or } 89 \text { ) }\end{array}$ & Glomerulonephritis \\
\hline 16 & Crescentic (extracapillary) glomerulonephritis (type I, II, III) & Glomerulonephritis \\
\hline 17 & Focal segmental glomeruloscerosis with nephrotic syndrome in adults & Glomerulonephritis \\
\hline 19 & Glomerulonephritis; histologically examined, not given above & Glomerulonephritis \\
\hline 20 & Pyelonephritis - cause not specified & Pyelonephritis \\
\hline 21 & Pyelonephritis associated with neurogenic bladder & Pyelonephritis \\
\hline 22 & Pyelonephritis due to congenital obstructive uropathy with/without vesico-ureteric reflux & Pyelonephritis \\
\hline 23 & Pyelonephritis due to acquired obstructive uropathy & Pyelonephritis \\
\hline 24 & Pyelonephritis due to vesico-ureteric reflux without obstruction & Pyelonephritis \\
\hline 25 & Pyelonephritis due to urolithiasis & Pyelonephritis \\
\hline 29 & Pyelonephritis due to other cause & Pyelonephritis \\
\hline 30 & $\begin{array}{l}\text { Interstitial nephritis (not pyelonephritis) due to other cause, or unspecified (not mentioned } \\
\text { above) }\end{array}$ & Interstitial \\
\hline 31 & Nephropathy (interstitial) due to analgesic drugs & Interstitial \\
\hline 32 & Nephropathy (interstitial) due to cis-platinum & Interstitial \\
\hline 33 & Nephropathy (interstitial) due to cyclosporin A & Interstitial \\
\hline 34 & Lead induced nephropathy (interstitial) & Interstitial \\
\hline 39 & Drug induced nephropathy (interstitial) not mentioned above & Interstitial \\
\hline 40 & Cystic kidney disease - type unspecified & Cystic/poly \\
\hline 41 & Polycystic kidneys; adult type (dominant) & Cystic/poly \\
\hline 42 & Polycystic kidneys; infantile (recessive) & Cystic/poly \\
\hline 43 & Medullary cystic disease; including nephronophtisis & Other \\
\hline 49 & Cystic kidney disease - other specified type & Other \\
\hline 50 & Hereditary/Familial nephropathy - type unspecified & Other \\
\hline 51 & Hereditary nephritis with nerve deafness (Alport's Syndrome) & Other \\
\hline 52 & Cystinosis & Other \\
\hline 53 & Primary oxalosis & Other \\
\hline 54 & Fabry's disease & Other \\
\hline 59 & Hereditary nephropathy - other specified type & Other \\
\hline 60 & Renal hypoplasia (congenital) - type unspecified & Other \\
\hline 61 & Oligomeganephronic hypoplasia & Other \\
\hline 63 & Congenital renal dysplasia with or without urinary tract malformation & Other \\
\hline 66 & Syndrome of agenesis of abdominal muscles (Prune Belly) & Other \\
\hline 70 & Renal vascular disease - type unspecified & Renal vascular disease \\
\hline 71 & Renal vascular disease due to malignant hypertension & Renal vascular disease \\
\hline
\end{tabular}




\begin{tabular}{lll}
\hline Code & Title & Group \\
\hline 72 & Renal vascular disease due to hypertension & Renal vascular disease \\
73 & Renal vascular disease due to polyarteritis & Renal vascular disease \\
74 & Wegener's granulomatosis & Other \\
75 & Ischaemic renal disease/cholesterol embolism & Renal vascular disease \\
76 & Glomerulonephritis related to liver cirrhosis & Other \\
78 & Cryoglobulinemic glomerulonephritis & Other \\
79 & Renal vascular disease - due to other cause (not given above and not code 84-88) & Renal vascular disease \\
80 & Type 1 diabetes with diabetic nephropathy & Diabetes \\
81 & Type 2 diabetes with diabetic nephropathy & Diabetes \\
82 & Myelomatosis/light chain deposit disease & Other \\
83 & Amyloid & Other \\
84 & Lupus erythematosus & Other \\
85 & Henoch-Schoenlein purpura & Other \\
86 & Goodpasture's Syndrome & Other \\
87 & Systemic sclerosis (scleroderma) & Other \\
88 & Haemolytic Ureaemic Syndrome (including Moschcowitz Syndrome) & Other \\
89 & Multi-system disease - other (not mentioned above) & Other \\
90 & Tubular necrosis (irreversible) or cortical necrosis (different from 88) & Other \\
91 & Tuberculosis & Other \\
92 & Gout nephropathy (urate) & Other \\
93 & Nephrocalcinosis and hypercalcaemic nephropathy & Other \\
94 & Balkan nephropathy & Other \\
95 & Kidney tumour & Other \\
96 & Traumatic or surgical loss of kidney & Other \\
98 & Not known & Missing \\
99 & Other identified renal disorders & Other \\
199 & Code not sent & Missing \\
\hline & &
\end{tabular}

\section{G3: EDTA cause of death}

\begin{tabular}{ll}
\hline EDTA code & Cause \\
\hline 0 & Cause of death uncertain/not determined \\
11 & Myocardial ischaemia and infarction \\
12 & Hyperkalaemia \\
13 & Haemorrhagic pericarditis \\
14 & Other causes of cardiac failure \\
15 & Cardiac arrest/sudden death; other cause or unknown \\
16 & Hypertensive cardiac failure \\
17 & Hypokalaemia \\
18 & Fluid overload/pulmonary oedema \\
21 & Pulmonary embolus \\
22 & Cerebro-vascular accident, other cause or unspecified \\
23 & Gastro-intestinal haemorrhage (digestive) \\
24 & Haemorrhage from graft site \\
25 & Hameorrhage from vascular access or dialysis circuit \\
26 & Haemorrhage from ruptured vascular aneurysm (not code 22 or 23) \\
27 & Haemorrhage from surgery (not codes 23, 24, 26) \\
28 & Other haemorrhage, (not codes 23-27) \\
29 & Mesenteric infarction \\
31 & Pulmonary infection bacterial (not code 73) \\
32 & Pulmonary infection (viral) \\
33 & Pulmonary infection (fungal or protozoal; parasitic) \\
34 & Infections elsewhere except viral hepatitis \\
&
\end{tabular}




\begin{tabular}{|c|c|}
\hline EDTA code & Cause \\
\hline 35 & Septicaemia \\
\hline 36 & Tuberculosis (lung) \\
\hline 38 & Generalized viral infection \\
\hline 39 & Peritonitis (all causes except for Peritoneal Dialysis) \\
\hline 41 & Liver disease due to hepatitis B virus \\
\hline 44 & Cirrhosis - not viral (alcoholic or other cause) \\
\hline 45 & Cystic liver disease \\
\hline 46 & Liver failure - cause unknown \\
\hline 51 & Patient refused further treatment for ESRF \\
\hline 52 & Suicide \\
\hline 53 & ESRF treatment ceased for any other reason \\
\hline 64 & Cachexia \\
\hline 66 & Malignant disease in patient treated by immunosuppressive therapy \\
\hline 67 & Malignant disease: solid tumors except those of 66 \\
\hline 68 & Malignant disease: lymphoproliferative disorders (Except 66) \\
\hline 69 & Dementia \\
\hline 70 & Peritonitis (sclerosing, with peritoneal dialysis) \\
\hline 71 & Perforation of peptic ulcer \\
\hline 72 & Perforation of colon \\
\hline 73 & Chronic obstructive pulmonary disease \\
\hline 81 & Accident related to ESRF treatment (not 25) \\
\hline 82 & Accident unrelated to ESRF treatment \\
\hline
\end{tabular}

